\title{
Pleural amyloidosis mimicking malignant mesothelioma
}

\author{
Min Yeong Kim¹, Tae Jung Kim ${ }^{1}$, Joungho Han ${ }^{2}$, Bo Da Nam ${ }^{1}$, Kyung Soo Lee \\ ${ }^{1}$ Department of Radiology and Center for Imaging Science, Samsung Medical Center, Sungkyunkwan University School of \\ Medicine, Seoul, Korea \\ ${ }^{2}$ Department of Pathology, Samsung Medical Center, Sungkyunkwan University School of Medicine, Seoul, Korea
}

Received: February 8, 2017

Revised: April 28, 2017

Accepted: May 19, 2017

Corresponding author:

Tae Jung Kim

Department of Radiology and

Center for Imaging Science,

Samsung Medical Center,

Sungkyunkwan University

School of Medicine, 81 Irwon-ro,

Gangnam-gu, Seoul 06351, Korea

Tel: +82-2-3410-0715

E-mail: taejung.kim1@gmail.com

\begin{abstract}
Pleural involvement of amyloidosis is rare and usually manifested as persistent massive effusion. However, it may present as pleural nodules or plaques without massive effusion. A 55-year-old man was referred for abnormalities of chest radiography and the computed tomography (CT) exam revealed multiple pleural nodules without effusion in the left hemithorax. The radiologic impression was pleural malignancy, and whole body $18 \mathrm{~F}$-fluorodeoxyglucose positron emission tomography-CT exam was performed. Not only corresponding pleural nodules but also other areas along the left pleura were hypermetabolic, whereas no hypermetabolic lesions were noted outside of the thorax. Preoperative diagnosis was made as malignant mesothelioma. During left pleuropneumonectomy, multiple hard whitish nodules or plaques were found along the pleura, and which were proven to amyloidosis on frozen section. The patient underwent not left pleuropneumonectomy but folup. Because of difficulty of accurate diagnosis, we report the localized pleural amyloidosis for awareness of the disease.
\end{abstract}

Keywords: Amyloidosis; Pleura; Positron-emission tomography; Tomography, X-ray computed

\section{INTRODUCTION}

Amyloidosis is a group of diseases characterized by deposition of amyloid substance in various organs. Thoracic involvement of amyloidosis is not frequent, especially plueral involvement is extremely rare.

We herein report a rare case of pleural amyloidosis which confused with malignant mesothelioma.

\section{CASE REPORT}

A 55-year-old man was referred to our hospital for evaluation of abnormalities of chest radiograph. He was asymptomatic and had a history of pulmonary tuberculosis. He had been a 28- 

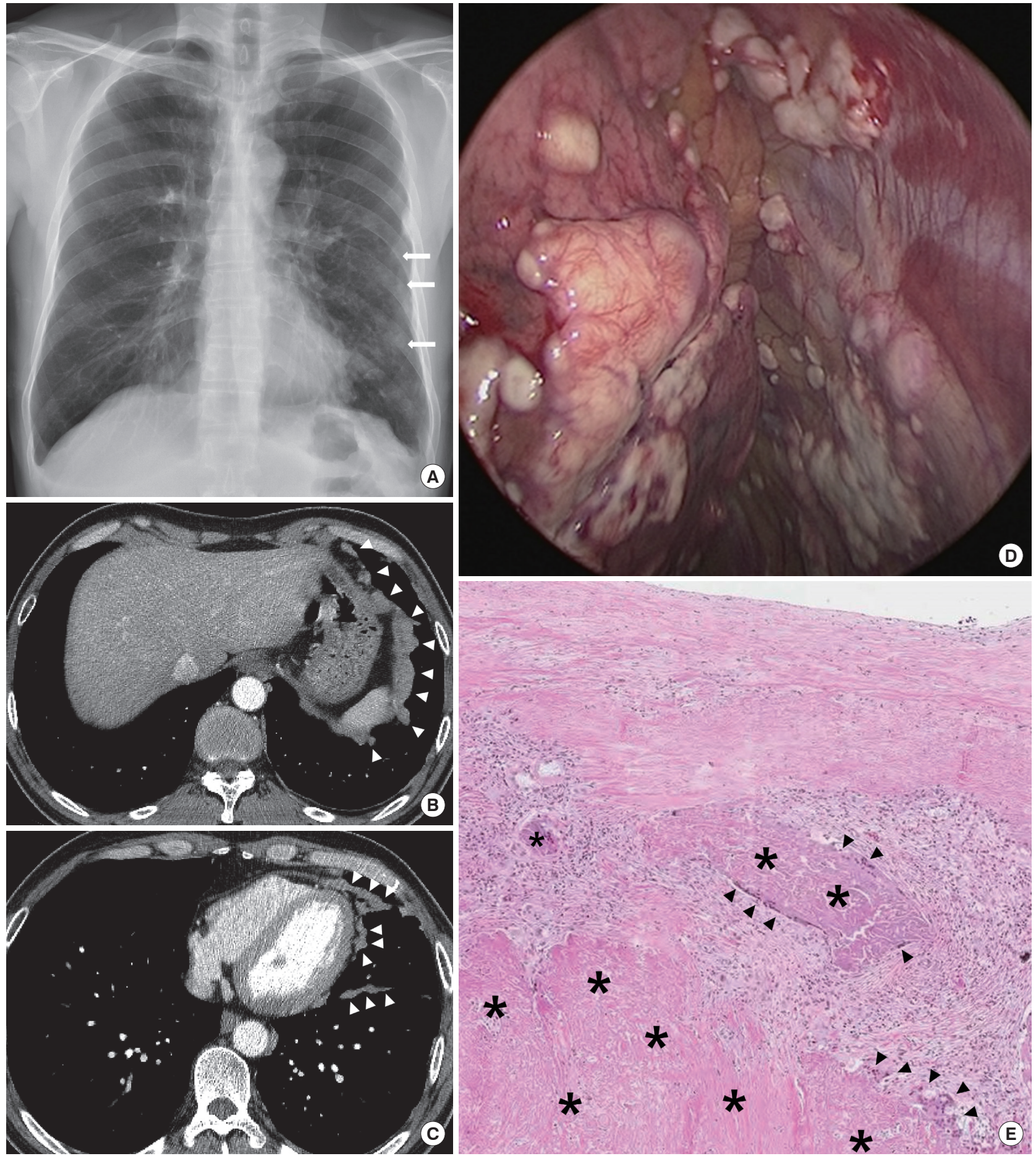

Fig. 1. A 55-year-old man with pleural amyloidosis mimicking pleural malignant mesothelioma. (A) Posteroanterior chest radiograph reveals multifocal ill-defined nodular densities (arrows) in left lower hemithorax. (B, C) Computed tomography (CT) scans demonstrate multifocal nodules along the diaphragmatic pleura and left major fissure (arrowheads) which are hypermetabolic on ${ }^{18} \mathrm{~F}$-fluorodeoxygloucose positron emission tomography-CT scans (not shown). Gross and microscopic findings of the pleural lesions in the left hemithorax. (D) There are multiple whitish nodules or plaques along the parietal pleura of diaphragm and chest wall. (E) On microscopic exam, amorphous eosinophilic materials (asterisks) are deposited among subpleural fibrotic tissue. Some multinucleated giant cells are surrounding these eosinophilic deposits (arrowheads) (H\&E, $\times 400)$. 
pack-year smoker but quit smoking 10 months ago. He was an engineer dealing with mobile networking systems. Physical exam, blood chemistry, and hematologic exams were not remarkable.

Chest radiography showed multiple ill-defined nodular lesions in left lower lung zone (Fig. 1A). Chest computed tomography $(\mathrm{CT})$ revealed multiple nodules along the diaphragmatic pleura and major fissure of left hemithorax. There was neither pleural effusion nor abnormalities in right hemithorax and mediastinum (Fig. 1B, C). Our primary radiologic impression was pleural malignancy, of which malignant mesothelioma was more probable than pleural metastasis. For evaluation of malignancy, whole body ${ }^{18} \mathrm{~F}$-fluorodeoxyglucose positron emission tomography $\left({ }^{18} \mathrm{~F}\right.$-FDG-PET)-CT exam was performed and hypermetabolic lesions, not only nodular lesions but also other areas along the left pleura, were noted. However there was no hypermetabolic lesion outside of the thorax. According to consensus of patient's physician, referring radiologists and thoracic surgeons, the patient was diagnosed as malignant mesothelioma and scheduled to undergo left pleuropneumonectomy.

In the surgical field, there were multiple variable-sized hard whitish nodules or plaques aggregating along the visceral and parietal pleura corresponding areas to preoperative CT scans (Fig. 1D). The surgeon requested intraoperative frozen section consultation to make a surgical decision. On microscopic exam, there were amorphous eosinophilic deposits which were positive of amyloid P component (Fig. 1E). The patient undergoes not left pleuropneumonectomy but follow up chest CT after 3 months, and which did not reveal any interval changes.

\section{DISCUSSION}

Amyloidosis is a group of diseases caused by the progressive deposition of amyloid protein in the extracellular spaces. Amyloid protein deposits always consist of three components: abnormally folded serum amyloid $P$, glycosaminoglycans and fibril proteins, which fold in a $\beta$-pleated sheet and characteristically retain the dye Congo-red. According to the type of the fibrillary component, the most important and major types are amyloid light (AL) chain and serum amyloid A (AA). $A L$ type is primary subtype and $A L$ protein is derived from the abnormal breakdown of normal immunoglobulin light chains in underlying plasma cell dyscrasia [1-3]. AA type is secondary subtype associated with systemic infection and inflammatory disorders, such as rheumatoid arthritis, chronic bron- chitis, and osteomyelitis [1].

In the thorax, amyloidosis can involve myocardium, mediastinal lymph nodes, and respiratory systems [1,2]. Incidence of respiratory amyloidosis is estimated at $28 \%$ of AL type but AL type accounts for $80 \%$ of respiratory amyloidosis [2,3]. There are four forms of respiratory amyloidosis, single or multiple nodular parenchymal amyloidosis, diffuse alveolar septal amyloidosis, airway amyloidosis, and pleural effusion [1,2]. Pleural effusion rarely occurs $[2,4,5]$. It can be persistent and massive not only by myocardial or renal amyloidosis but also diffuse pleural infiltration of amyloid [1-6]. Pleural effusion is diverse, transudative, exudative, and even hemorrhagic [2,46]. Thoracoscopic findings are characteristic, fine white or yellow nodules or granular lesions along parietal pleura $[2,4,5]$. However asymptomatic localized pleural amyloidosis is extremely rare and may present as multiple nodules or plaques along pleura without massive effusion [7-9]. Furthermore, amyloid lesions are usually hypermetabolic on FDG-PET scans, because of abnormal production of immunoglobulins by plasma cells $[8,9]$. Therefore localized pleural amyloidosis can be misdiagnosed as malignant mesothelioma or solitary fibrous tumor $[7,8]$. According to literature, intraoperative frozen section consultation was need in two of four proven cases of pleural amyloidosis $[7,8]$.

Including our case, all five cases of localized pleural amyloidosis has characteristic manifestation. The lesions were either nodules or plaques in limited area of pleura and pleural effusion was not associated [7-9]. Radiologic diagnosis of the cases were pleural malignancies except for one case [9]. After surgical resection, the lesions showed no progression or recurrence on follow-up imaging [7-9]. All five patients were male and did not have history of multiple myeloma, even though all pathologic subtypes were AL amyloidosis [7-9]. Because of the rarity of the pleural amyloidosis localized in unilateral thorax, accurate diagnosis might have been difficult for both radiologists and physicians.

In conclusion, localized pleural amyloidosis is extremely rare but can mimic pleural malignancy clinically and radiologically. Awareness of its unique manifestation is necessary and timely histologic confirmation must be considered for adequate treatment decision.

\section{CONFLICTS OF INTEREST}

No potential conflict of interest relevant to this article was reported. 


\section{ORCID}

Min Yeong Kim https://orcid.org/0000-0002-6228-7313

Joungho Han https://orcid.org/0000-0003-4424-7008

Bo Da Nam https://orcid.org/0000-0001-7822-6104

\section{REFERENCES}

1. Czeyda-Pommersheim F, Hwang M, Chen SS, Strollo D, Fuhrman C, Bhalla S. Amyloidosis: modern cross-sectional imaging. Radiographics 2015;35:1381-92.

2. George S, Ravindran M, Anandan PT, Kiran VN. Primary systemic amyloidosis: a rare cause for pleural effusion. Respir Med Case Rep 2014;13:39-42.

3. Berk JL, Keane J, Seldin DC, Sanchorawala V, Koyama J, Dember LM, et al. Persistent pleural effusions in primary systemic amyloidosis: etiology and prognosis. Chest 2003; 124:969-77.

4. Bontemps F, Tillie-Leblond I, Coppin MC, Frehart P, Wal- laert B, Ramon P, et al. Pleural amyloidosis: thoracoscopic aspects. Eur Respir J 1995;8:1025-7.

5. Mansalis KA, Klein DA, Demartini SD, Powers JF, Danielson DS. Pleural findings in a patient with persistent pulmonary effusions from systemic amyloidosis. Amyloid 2011;18:29-31.

6. Shimizu Y, Endou K, Hashizume Y, Kawata T, Horie T, Ogu$\mathrm{ra} \mathrm{H}$, et al. Massive pleural effusion due to pleural AA amyloidosis. Am J Respir Crit Care Med 2012;186:e19-20.

7. Adams AL, Castro CY, Singh SP, Moran CA. Pleural amyloidosis mimicking mesothelioma: a clinicopathologic study of two cases. Ann Diagn Pathol 2001;5:229-32.

8. Nakano T, Endo S, Tetsuka K, Fukushima N. Asymptomatic localized pleural amyloidosis mimicking malignant pleural mesothelioma: report of a case. J Thorac Dis 2016;8: E157-60.

9. Yoshiya S, Maruyama R, Koga T, Shikada Y, Yano T, Maehara Y. Localized pleural amyloidosis: report of a case. Surg Today 2012;42:597-600. 